\title{
Propagation properties of Electron Vortex Beams
}

\author{
K. Saitoh ${ }^{1}$, H. Nambu ${ }^{2}$, N. Tanaka ${ }^{1}$ and M. Uchida ${ }^{3}$ \\ ${ }^{1}$ EcoTopia Science Institute, Nagoya University, Nagoya 464-8603, Japan \\ ${ }^{2}$ Department of Crystalline Materials Science, Nagoya University, Nagoya 464-8603, Japan \\ ${ }^{3}$ Advanced Science Research Laboratory, Saitama Institute of Technology, Fukaya 369-0293, Japan
}

Electron vortex beam, an electron beam carrying an orbital angular momentum (OAM), has been attracting a great attention by its unique physical property and application to a new microscopy in materials science $[2,3]$. In the present paper, we show our latest studies on the generation and propagation dynamics of electron vortex beams $[4,5,6]$.

Electron vortex pairs embedded in a single beam are generated using a nanofabricated holographic grating and observed their propagation dynamics. Figure 1(a) shows successive series of cross sections of a propagating electron beam which has two vortices with the same topological charge of +1 . The pair of the phase singularities, which are indicated by arrows, rotates in the same direction as the beam propagates. When the topological charges of the two vortices are opposite to each other, they rotate opposite direction. The experimental results are well explained by the Gouy phase shift and confirmed by numerical simulations of the propagation. The results of this study may help develop an intuitive understanding of electron vortex motion and give a new viewpoint for analyzing electron microscope images and phase change in a crystal.

We seek to explore the measurement of electron OAM by using a nano-fabricated, forked grating. We experimentally examine how an electron vortex beam with orbital angular momentum (OAM) undergoes diffraction through a forked grating. The $n$ th-order diffracted electron vortex beam after passing through a forked grating with a Burgers vector of 1 shows an OAM transfer of $n \hbar$. Hence, the diffraction patterns become mirror asymmetric owing to the size difference between the electron beams. Such a forked grating, when used in combination with a pinhole located at the diffraction plane, could act as an analyzer to measure the OAM of input electrons [6]. This may explain the results of recent experimental studies by Verbeeck et al., in which the observation of dichroism is reported in EELS of ferromagnetic Fe thin films using electron vortex beams [2].

We also study the utility of forked gratings for phase retrieval. Forked gratings produce diffracted peaks of electron vortex beams. These peaks have rich information of phase distribution on a plane of the forked grating. We apply the Fourier iterative phase retrieval technique for diffraction patterns obtained using forked gratings. Figures 2(a) and 2(b) show two examples of phase retrievals. Fig. 2(a) shows a phase retrieval of a forked grating without any specimen. The phase retrieval shows a good convergence and robustness compared to that done by a simple circular aperture, indicating that the diffracted vortex peaks carry additional information for the phase retrieval. A simulation study shows a correlation between the specimen shape and distortion manner of the diffracted electron vortex beams, which might be used to map a phase distribution in real space. 

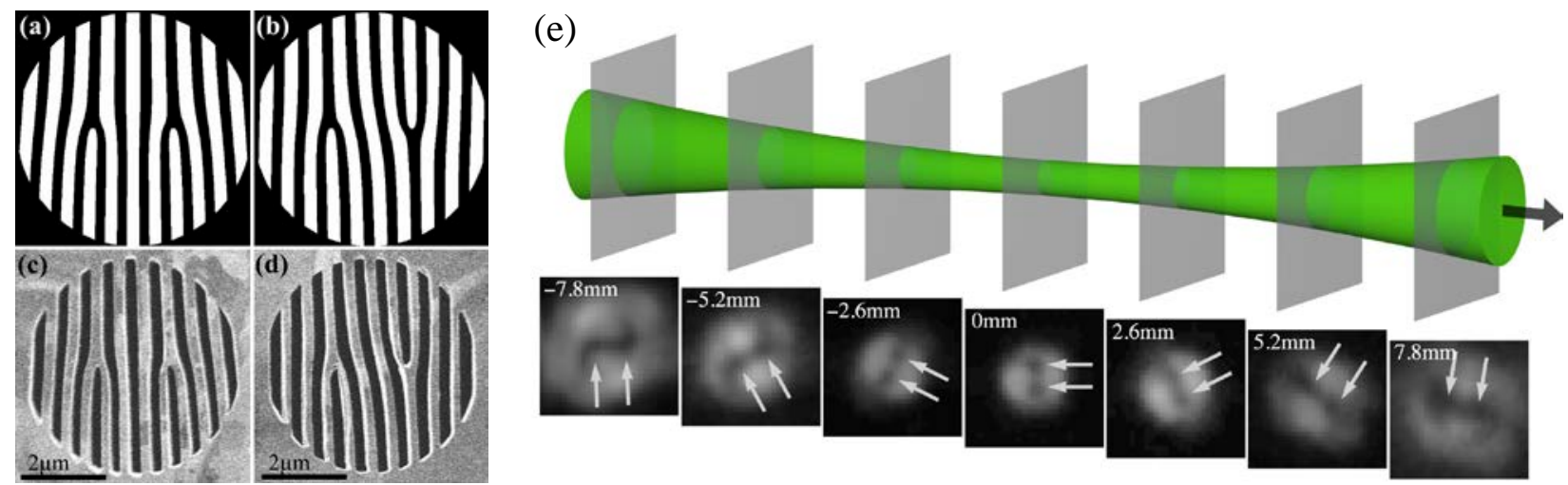

Fig. 1. Forked gratings generating Electron vortex pairs embedded in a single beam. Binarized computer holograms calculated with topological charges of $(+1,+1)(a)$ and $(+1,-1)(b)$. Electron beam masks of $(+1,+1)(\mathrm{c})$ and $(+1,-1)(\mathrm{d})$, which are nano-fabricated by a focused ion beam instrument. (e) A successive series of cross sections of a propagating vortex pair embedded in a single beam.
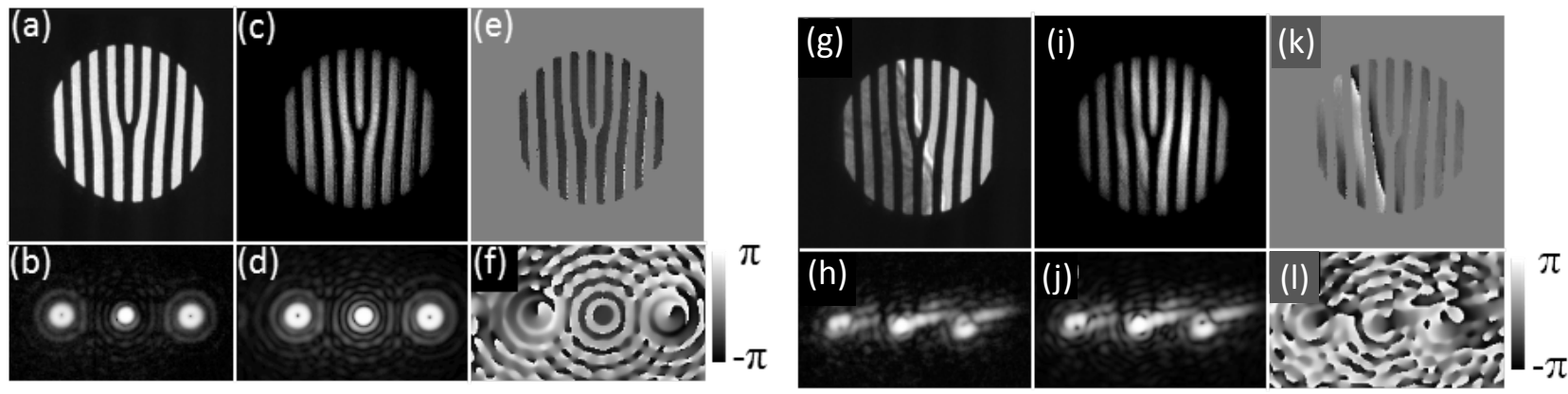

Fig. 2 (a)-(f): Phase retrieval of a forked grating without specimen. Experimental TEM image of forked grating (a) and diffraction pattern of (b) are used as real space and reciprocal space constraints. The amplitudes of the image (c) and diffraction pattern (d) are well reproduced by the present analysis. A constant phase in the image (e) and phase rotation of each diffracted peaks in the diffraction pattern are visualized. (g)-(l): Phase retrieval of a forked grating with a specimen of a Fe thin film. The introduction of the specimen disturbs circular symmetric vortex peaks. The amplitudes of the experimental image (g) and diffraction pattern (h) are well reproduced by the present analysis as shown in (i) and (j), respectively.

\section{References}

[1] M. Uchida and A. Tonomura, Nature 464 (2010) 737.

[2] J. Verbeeck, H. Tian, and P. Schattschneider, Nature 467 (2010) 301.

[3] B. J. McMorran et al. Science 331 (2011) 192.

[4] K. Saitoh et al. J. Electron Microsc. 61 (2012) 171.

[5] Y. Hasegawa et al. J. Phys. Soc. Jpn. 82 (2013) 033002.

[6] K. Saitoh et al, Phys. Rev. Lett. (2013). 